\title{
Emergent Space-Time via a Geometric Renormalization Method
}

\author{
Saeed Rastgoo $1, *$ and Manfred Requardt ${ }^{2, \oplus}$ \\ ${ }^{1}$ Departamento de Física, Universidad Autónoma Metropolitana - Iztapalapa \\ San Rafael Atlixco 186, Mexico D.F. 09340, Mexico \\ ${ }^{2}$ Institut für Theoretische Physik Universitaet Goettingen \\ Friedrich-Hund-Platz 1, 37077 Goettingen, Germany
}

(Dated: December 19, 2016)

\begin{abstract}
We present a purely geometric renormalization scheme for metric spaces (including uncolored graphs), which consists of a coarse graining and a rescaling operation on such spaces. The coarse graining is based on the concept of quasi-isometry, which yields a sequence of discrete coarse grained spaces each having a continuum limit under the rescaling operation. We provide criteria under which such sequences do converge within a superspace of metric spaces, or may constitute the basin of attraction of a common continuum limit, which hopefully, may represent our space-time continuum.

We discuss some of the properties of these coarse grained spaces as well as their continuum limits, such as scale invariance and metric similarity, and show that different layers of spacetime can carry different distance functions while being homeomorphic.

Important tools in this analysis are the Gromov-Hausdorff distance functional for general metric spaces and the growth degree of graphs or networks. The whole construction is in the spirit of the Wilsonian renormalization group.

Furthermore we introduce a physically relevant notion of dimension on the spaces of interest in our analysis, which e.g. for regular lattices reduces to the ordinary lattice dimension. We show that this dimension is stable under the proposed coarse graining procedure as long as the latter is sufficiently local, i.e. quasi-isometric, and discuss the conditions under which this dimension is an integer. We comment on the possibility that the limit space may turn out to be fractal in case the dimension is non-integer. At the end of the paper we briefly mention the possibility that our network carries a translocal far-order which leads to the concept of wormhole spaces and a scale dependent dimension if the coarse graining procedure is no longer local.
\end{abstract}

\footnotetext{
* saeed@xanum.uam.mx

$\dagger$ requardt@theorie.physik.uni-goettingen.de
} 


\section{CONTENTS}

I. Introduction

II. Main strategy: the big picture

III. Macro States, Micro States, and Typical States 9

IV. Graphs as metric spaces $\quad 10$

$\mathrm{V}$. A generic coarse graining scheme 11

A. Some concrete examples of coarse graining $\mathcal{K}$

1. Quasi-isometry coarse graining 14

$k$-local edge insertion/deletion 14

Vertex contraction of diameter $\leq k$

2. Rough isometry coarse graining 14

Clique graph transformation $\mathcal{C}(G)$

VI. The Gromov-Hausdorff Space of Metric Spaces

VII. Convergence and Continuum Limit I 18

\begin{tabular}{l|l|} 
VIII. Convergence and Continuum Limit II & 19 \\
\hline
\end{tabular}

IX. The Geometric Renormalization Group in the Superspace of Metric Spaces 21

X. Dimension

XI. Conclusion $\quad 25$

$\begin{array}{ll}\text { Acknowledgments } & 27\end{array}$

A. Some relevant definitions

B. Proof of some of the theorems

Theorem V.3

Theorem IX.7

References $\quad[29$

\section{INTRODUCTION}

Classical macroscopic space-time (henceforth: S-T) is a continuous manifold on macroscopic scales. The physical (quantum) fields live on this continuous manifold as separate entities. Macroscopic objects move freely through S-T. On the other hand, on a more microscopic (but, compared to the infamous Planck scale, still mesoscopic) scale the quantum vacuum appears to be full of quantum fluctuations while on a still finer scale even S-T itself is expected to wildly fluctuate. 
While in string theory the framework is (at least initially) constructed over smooth (higher dimensional) manifolds, in most of the other approaches to quantum gravity one assumes that at a primordial level S-T is both discrete and presumably quite erratic. A crucial concept in these latter approaches is the notion of background independence.

There have been various attempts to introduce a discrete structure and reconstruct smooth classical (or macroscopic) S-T from such a discrete and irregular substratum (we will discuss in more detail in the following section what is meant by these attributes). But as this paper is not intended to be a review we are able to mention only a few randomly selected sources. Examples are the spin networks and spin foam models in loop quantum gravity (LQG) and its path integral versions. Some more recent examples are the quantum graphity approach [1-12], the group field theory framework and the random tensor networks [13, 14]. A nice description of the whole field can for example be found in [15] with emphasis on the emergence of space-time in the various approaches.

All these approaches are, to a larger or lesser degree, derived from attempts to directly quantize classical general relativity (GR). The same holds for dynamical triangulation and related frameworks like e.g. Regge calculus [16]. Another approach has been developed by ourselves and coworkers and is based on generalizations of cellular automata (CA), i.e. Structurally Dynamic Cellular Networks (SDCN); for more information see the recent review [17]. To this class also belongs e.g. [18] which studies random-Ising-like models of spacetime. One should also mention the work in general network theory, see e.g. the recent [19] or our paper [20]

The point of view, shared by all these approaches is the conviction that $\mathrm{S}-\mathrm{T}$ on its most primordial level is a dynamic substratum consisting of certain elementary degrees of freedom. Their nature, however, may be different in the various schools. In many frameworks they carry a certain a priori geometric flavor (inspired by the simplicial resolution of continuous manifolds) and consist for example of infinitesimal triangles or tetrahedrons having, a fortiori, edges of a certain infinitesimal length. In other approaches these microscopic degrees of freedom (DoF) are viewed more abstractly as elementary cells, carrying internal states, interacting with each other (or exchanging information) via elementary interactions. In this latter case the quantum vacuum is regarded as a huge irregular dynamical system (e.g. the SDCN). The geometric notions are here considered to emerge from a primarily non-geometric substratum in the spirit of J. A. Wheeler: Geometry from Non-Geometry (see Sect. 44.4 of [21]). In e.g. LQG the elementary DoF carry both qualities to a greater or lesser degree, i.e. geometric ones and more abstract ones. Another important point: the DoF may not even have any local character. The localization of objects on coarser scales may also be emergent in a relational way.

In concluding our brief discussion of the various points of view expressed in the frameworks addressed above we want to comment on some remarks made in the nice contribution of Bombelli et. al. [22] because it offers us the opportunity to clarify some points of principle and misunderstandings. This paper deals mostly with the reconstruction of a continuum manifold via a piecewise linear (embedded) manifold which is, on its side, derived from certain graphs. This they call the inverse problem.

One should say that this is a fairly widespread strategy in the mathematics of manifolds with a huge amount of published results. An important question in fundamental physics is to what extent nature on its most primordial level is actually concerned with such geometric micro objects like simplices, tetrahedra and the like.

We shall argue in the following that quantum space-time is, in our view, rather an ex- 
tremely complex and erratic dynamical system, consisting of an array of elementary DoF together with elementary interaction among these DoF. It is then the task to derive geometric notions (and for example continuum analysis) from such a primordial substratum. We developed such concepts in e.g. [23] as well as a certain discrete calculus (which has relations to non-commutative geometry). We showed for example that one can develop a kind of (co)homology theory (see section 3.2) by associating simplices to subsets of DoF with elementary interactions existing between all the respective pairs of DoF in the subset. For more information see our recent [17].

To clear things up a bit regarding some of the comments in [22] about the notion of the dimension introduced in [24], we mention a few words. We developed this concept of dimension for graphs and networks in [25], being mainly motivated by physical ideas. We studied how space dimension really enters in the physical formulas in say statistical mechanics, critical phenomena etc. We realized that what typically really matters is the number of new interaction partners a local site sees after consecutive steps on e.g. a lattice, embedded in some continuous space. It then happens that the dimension of the embedding space enters in a characteristic way in the physical formulas. We observed later in [24] that a related notion was used in a beautiful field of pure mathematics, that is, geometric group theory, viz. Cayley graphs and is there called the growth degree.

One should furthermore mention that our concept of dimension has, despite its superficial similarity, nothing to do with any fractal dimension. The latter concept describes the behavior of a system in the infinitely small while our (scaling) dimension rather characterizes the large scale properties of graphs and networks and is an important invariant of such structures. Its advantage is that it shows that integer dimensions are very particular while non-integer dimensions are rather the rule (as in the fractal case). This gives us a tool and criterion to single out spaces having an integer macro-dimension (like our own S-T). On the other hand, many of the more geometric approaches are dealing right from the start with integer dimensions. We think, it may be interesting to learn that the integer dimensionality of our physical S-T needs some explanation.

As we already indicated, our aim in the following is it to develop a coarse graining scheme and continuum limit comprising as many different frameworks as possible. One should note, however, that this is an ambitious task as the meaning of coarse graining and/or continuum limit may have a different meaning in the various approaches. The same holds for the concept of dynamics (we comment on these points in the next section). To this end we make the following assumption:

Assumption I.1. We assume that $S$ - $T$ on its most primordial level is a complex dynamical system, consisting of a huge array of microscopic DoF together with a (random) distribution of elementary interactions connecting these DoF.

One should perhaps emphasize that working with networks or graphs does not mean that we really think of the most primordial objects as extensionless points, quite to the contrary, they are usually assumed to represent certain lumps having an internal structure which, on the respective scale of resolution, cannot be resolved (cf. our paper [26] and the remarks by Menger at the end of his contribution in [27]). Our following contribution shows that Einstein's skeptical remarks concerning such a radical program made in [27] was perhaps too conservative.

As a further remark, there is yet another approach towards a space of spaces in (quantum) space-time physics. i.e. classifying space structure via the spectrum of the Laplacian [28], but we do not touch this field in this paper. 
The structure of the paper is as following: we start in section II by presenting the big picture and main steps of the work, so that the reader does not get lost in the details of the following sections. In section III, we present the idea of a phase cell in state space of a system, that can be considered as the basin of attraction of the evolution map. This will be used to categorize the class of states (discrete spaces) that yield the same smooth continuum limit. Section IV contains necessary concepts about the graphs as metric spaces. In section $[\nabla$ we present our generic coarse graining schemes, consisting of quasi-isometry and rough isometry, and provides some examples of each. In section VI, we introduce the Gromov-Hausdorff space, and its associated metric, the Gromov-Hausdorff metric with respect to which (non)isometry of spaces is measured. Sections VII and VIII are devoted to the notions of convergence of a sequence of metric spaces, and defining their rescaling and continuum limit. In section IX, we combine all the information in previous sections and fully develop and describe the geometric renormalization process that can lead from a discrete structure like a graph to a continuum limit such as a manifold. In section $\mathrm{X}$ we present a notion of dimension in our spaces, and very briefly discuss its properties under the geometric renormalization process. Finally, we summarize and make some concluding remarks in section XI. The appendix includes several definitions that are needed for the paper to be self-consistent.

\section{MAIN STRATEGY: THE BIG PICTURE}

Any theory with a discrete or quantum pregeometry, is faced with the challenge of deriving a continuum limit for this discrete structure, that looks like a desired smooth spacetime manifold. Of course we are aware of the facts that discrete does not always mean quantum, and the concepts of semiclassical limit and continuum limit are, in most cases, not the same, describe different physics and do not commute.

Remark II.1. We would like to clarify what we mean by a continuum limit. In various approaches it means simply a way of embedding a discrete (approximate) structure into some (preexisting) background manifold as in so called piece-wise linear geometry. Our enterprise is much more ambitious. We rather perform a true scaling limit starting from a sequence of discrete spaces. That is, the limit space can have a quite complicated continuous structure.

The semiclassical limit is generally associated to large quantum numbers, examples of which can be found in ferromagnetism and superconductivity, which in some cases may be seen as a manifestation of the correspondence principle. In loop quantum gravity for example, semiclassical typically refers to a limit of a fixed finite (generally small) number of large quantum numbers (spins) corresponding to a graph on which a coherent state (labelled by the aforementioned spins) lives. This semiclassical limit on large scales, evidently corresponds to the Regge-gravity.

The continuum limit on the other hand may be seen as being related to the so called thermodynamic limit, where there is a huge (or infinite) number of DoF each with small associated quantum numbers. In LQG, for example, the continuum limit generally refers to many, perhaps infinite, number of small spins.

Also there may be states in the theory that may not have any semiclassical counterpart, such as the replacement of cosmological singularities with highly quantum nonsingular structures in loop quantum cosmology [29]. 
There are many viewpoints and methods that deal with the issue of getting spacetime (or discrete/continuous general relativity) as their semiclassical or continuum limit. In what follows we try to make some clarifying remarks regarding our method and its relation to some of other ones. We begin with some brief remarks how a continuum limit is understood in LQG as presented in [11, 12] which refers there to [1]. There, the ultimate task is to construct a continuum physical Hilbert space, satisfying the constraints of the theory, as a limit of an inductive process, associated to iterated refinements of graphs embedded in a spatial manifold.

What we have in mind, on the other hand, is rather a more direct way of constructing a kind of a macroscopic continuous smooth manifold, representing the classical spacetime, out of a discrete fundamental structure (a graph). This transition from a graph to a smooth structure is implemented with the help of two different kinds of operations. One of these operations is, in a sense, related to the coarse graining within discrete structures. The second one, i.e. a rescaling operation, leads to a macroscopic continuum (in the optimum case). It defines the true transition from discrete spaces to continuous spaces and is quite non-trivial.

Another point to be briefly discussed is the kind of structures being constructed over this discrete network. In canonical LQG, the spin networks, constituting a complete basis of the Hilbert space of the solutions to the quantum Gauss constraint, are represented by graphs whose edges are colored by the irreducible representations of a compact group $(S U(2))$, and whose vertices are the intertwiners of the representations of the edges that are connected to those vertices. In our own approach, inspired by the generalization of cellular automata, the elementary structure also consists of vertices with simple internal states (and like LQG obey some kind of consistency relation based on the states of the edges connected to them), and edges which can change their orientation (directed graphs) or be deleted or created due to the dynamics. A choice of the dynamics can be given by an interaction of edge and vertex states yielding a new network, after each evolution step called a "clocktime" step. As in the spin network case we can define Hilbert spaces over the vertices and edges and then graph operators such as discrete Laplacians and Dirac operators. This procedure also establishes a connection to Connes' noncommutative geometry (for details see for example [23], [30] or [31]). In section 4 of [32] we showed that the evolving dynamics belongs to the same general class of graph transformations or dynamics, as is the case for spin network dynamics or causal set dynamics. I.e., we discuss in this paper primarily the continuum limit of the "space-like slices" in $S-T$. The dynamics is formulated as for spin-networks or causal sets by having a consecutive sequence of such geometric networks states given by some graph transformation rule. For convenience we prefer to call our underlying substratum $S-T$ while, for the time being, we mainly deal with the $S$-part.

Furthermore, in [33] we performed large scale numerical computer studies of various dynamical or statistical parameters of our networks to learn about their large scale or longtime behavior, or to find phase transitions. That is, we think all these various approaches, while being different with respect to their technical details, are on the other hand sufficiently related so that a joint treatment seems to be reasonable. We would like to resume what we are going to do and what we will not do:

Remark II.2. In the following we will abstract completely from the internal edge and vertex states, the Hilbert space structure and the operators living on the network as well as the dynamics, as we suspect that their respective macroscopic or continuum limit can be studied separately. We concentrate exclusively on the geometric state of our network, that is, its wiring diagram. We surmise that this purely geometric substratum and its evolution (as 
in the causal set approach) may describe the fine structure of our macroscopic space-time manifold.

It is clear that the network dynamics also changes after each coarse graining or renormalization step. In previous work (see e.g. [32] or our recent review [17]) we formulated for example a class of graph dynamics via an interaction of vertex and edge states which can be applied on each level of coarse graining. By the same token one can study the limit behavior of operators and fields defined on our networks but we will postpone this investigation to future work. By the way, one has to be prepared to deal with operators on fractal spaces which is no easy task.

A last remark concerning the generality of our approach. We think that the definition of coarse graining and/or renormalization via general quasi-isometries comprises many of the possible renormalization schemes, but we have not investigated every suggestion in detail.

We want to conclude this brief survey with a remark concerning the diffeomorphism invariance which is a central theme in the theory of gravitation and consequently in LQG. Without constantly mentioning it, it is also encoded in our own approach which is based on the theory of general metric spaces.

Remark II.3. In the following we usually deal with equivalence classes of metrically isomorphic spaces. That is, the limits we are going to construct consist of whole classes of isomorphic spaces which are treated as essentially undistinguishable. In more technical terms (to be defined in the following) they have Gromov-Hausdoff-distance zero. However, there may exist spaces with GH-distance zero, which are not isomorphic. That is, this class may be actually larger. In any case, this kind of equivalence could in our view be considered as being similar to diffeomorphism invariance.

Let us now start to develop the general framework of our approach (the main ideas and a large part of the work introduced here is based on [24]). As we mentioned earlier, this is a rather direct, and to a great extent, general approach, since it deals with general types of graphs, and does not assume any specific Hamiltonian, action, etc., for the semiclassical or continuous theory, although the final goal is derive an emergent smooth manifold equipped with a metric that satisfied general relativity. More specifically, we ask: given a graph $G_{0}$ (viz., the geometric abstraction of a network of interacting sites) as our pregeometric structure and a physically motivated coarse graining process, is it possible to get to a smooth manifold as the semiclassical limit of $G_{0}$ ? and if so, how? and under what conditions?

This of course depends on the coarse graining process, and on the criterion used to measure the convergence to a limit space which can possibly be associated to our space-time manifold. All of this will be discussed in detail in the following sections. To give a big picture of the strategy, here is how we attack the problem:

1. We consider the superspace $\mathcal{S}$ of all non-compact but locally compact metric spaces, including suitable uncolored graphs 1 and suitable manifolds with a metric. This can be seen to be an analogue of the "theory space" in renormalization methods.

2. A graph $\left(G_{0}, d_{0}\right) \in \mathcal{S}$, with $d_{0}$ being a graph metric, is chosen as our initial system, representing the fundamental layer that constitutes the fundamental discrete level of the smooth spacetime. To get to the smooth spacetime, two types of operations are introduced:

\footnotetext{
1 The treatment of colored graphs is postponed to future works.
} 
(a) A -to some extent- generic coarse graining scheme $\mathcal{K}$, based on the notion of quasi-isometry. This coarse graining procedure, assigns to a metric space (in this case a graph) $\left(G_{1}, d_{1}\right) \in \mathcal{S}$, another metric space $\left(G_{2}, d_{2}\right) \in \mathcal{S}$ such that $\left(G_{2}, d_{2}\right)$ is the coarse grained space derived from $\left(G_{1}, d_{1}\right)$ by applying $\mathcal{K}$ once:

$$
\left(G_{1}, d_{1}\right) \stackrel{\mathcal{K}}{\longrightarrow}\left(G_{2}, d_{2}\right) .
$$

This process can be performed repeatedly to give us a sequence of (possibly countably infinite number of ) coarse grained spaces $\left\{\left(G_{i}, d_{i}\right)\right\}, i=0, \ldots, N$ such that

$$
\left(G_{0}, d_{0}\right) \stackrel{\mathcal{K}}{\longrightarrow}\left(G_{1}, d_{1}\right) \stackrel{\mathcal{K}}{\longrightarrow}\left(G_{2}, d_{2}\right) \stackrel{\mathcal{K}}{\longrightarrow} \cdots \stackrel{\mathcal{K}}{\longrightarrow}\left(G_{N}, d_{N}\right),
$$

where all the $\left(G_{i}, d_{i}\right)$ 's belong to $\mathcal{S}$. We call this chain, a coarse graining chain.

(b) A rescaling map

$$
\phi_{\lambda}:\left(G_{i}, d_{i}\right) \longmapsto\left(G_{i}, \lambda d_{i}\right)
$$

on each member of the coarse graining chain, such that the limit $\lambda \rightarrow 0$ of the above map corresponds to the continuum limit $\left(G_{i, \infty}, d_{i, \infty}\right)$ of $G_{i}$,

$$
\lim _{\lambda \rightarrow 0} \phi_{\lambda}\left(\left(G_{i}, d_{i}\right)\right)=\left(G_{i, \infty}, d_{i, \infty}\right) .
$$

3. Combining the two above operations, the following picture arises

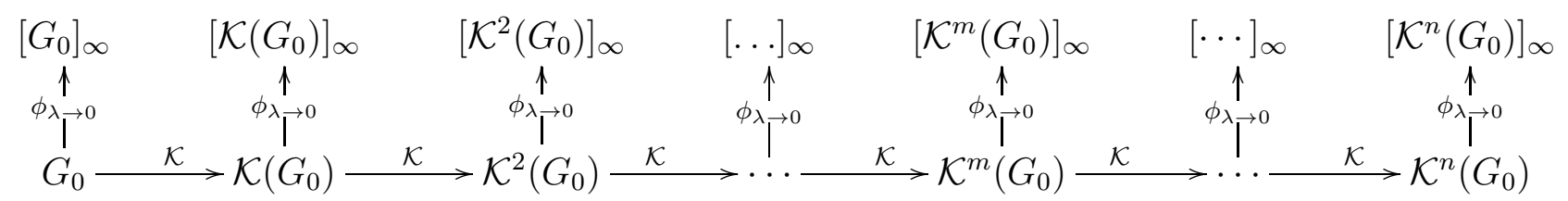

where $\left[\mathcal{K}^{i}\left(G_{0}\right)\right]_{\infty}=G_{i, \infty}$. In the coarse graining chain of discrete spaces (the lower horizontal chain), the coarse graining operation $\mathcal{K}$ is applied consecutively until one (or possibly both) of the followings happens:

(a) At a certain point the spaces become roughly isometric (that is, $\mathcal{K}$ makes a transition from being a true quasi-isometry to a rough isometry). In this case we show that the continuum limits of this row of roughly isometric spaces are the same continuous space.

(b) The coarse graining chain ends in a fixed point or a set of accumulation points under $\mathcal{K}$. This happens after a finite number of steps. We think however that this is a non-generic situation. For more remarks see section [X]

4. Before becoming roughly isometric, the memebers of the coarse graining chain are purely quasi-isometric. In this case we show that the scaling limits $G_{i, \infty}, G_{j, \infty}$ of two such spaces $G_{i}$ and $G_{j}$ are homeomorphic (even can be chosen to be the same topological space) but carry different metrics. This implies that different levels of spacetime can have different metric even if they are the same set.

5. Finally we define a notion of dimension, and briefly discuss the conditions on its integerness, its stability, or change under the coarse graining and scaling operations. 
The whole process prehaps resembles the idea of the renormalization group in the Wilsonian sense. This is of course not accidental. In [32] we called a certain coarse graining scheme the geometric renormalization group. We will complement it with a rescaling process towards some continuum space developed in [24].

Before continuing, we should mention that some of the important concepts are written in bold, and for self-containment, many of them are either defined in the main body of the text, or are presented in the appendix.

\section{MACRO STATES, MICRO STATES, AND TYPICAL STATES}

It is common practice in the physics of large and complicated systems, consisting of a huge number of interacting microscopic DoF, to work with ensembles of states or configurations. The same is the case in the various approaches to quantum gravity. Frequently something like a canonical ensemble over micro states is used, the statistical weight being given by a (pseudo) Hamiltonian, the main purpose of which is usually defining the exponent in the Boltzmann weight. One should note, however, that it is not completely clear if the concept of a Hamiltonian, apart from its mere probabilistic role, is an adequate notion in fundamental space-time physics.

On the other hand, we observe that macroscopic space-time, as the stage on which all the usual physical processes are going on, is not some ensemble in our various model theories. In the following we want to briefly explain how we see its role in our paper.

In [34] we indicated that S-T may rather be called an order parameter manifold with the non-vanishing metric tensor $g(x)$ being an order parameter field. The concept of an order parameter stems from the statistical mechanics of phase transitions and we think, a similar phenomenon can be seen in our space-time context if we view S-T as described in the introduction. We plan to give more details elsewhere, for the time being we content ourselves to invoke the following picture:

Conjecture III.1. Classical space-time, S-T, is the macroscopic, long-distance, low-energy description of an ensemble of microscopic configurations of only incompletely known (quantum) nature. As in statistical mechanics, we regard this ensemble of underlying micro states as a phase cell of states which look alike macroscopically.

To our knowledge, such a dual structure was for the first time analyzed by v. Neumann in a beautiful paper [35] in the context of the quantum theory of many DoF. It was recently translated into English and commented upon in [36]. A particular role is played by the emergence of commuting macro observables, a topic which was further developed in [37]. We think, the situation in (quantum) space-time physics is very much the same ( Recently this kind of typicality was also studied in our context in e.g. [38] and [39]).

In a next step we want to argue why we nevertheless are allowed to deal in our investigation with single states, thus avoiding the intricate study of ensembles of micro states describing S-T on microscopic scales, and in particular, their behavior under coarse graining. This point was also discussed for the first time in [35], for a recent discussion see e.g. [40]. Without giving proofs (a more systematic study can be found in [41], some examples are also discussed in the famous section 3.5 of [42]) we would like to state the following:

Observation III.2. It is an important observation that many systems consisting of a great number of microscopic DoF display the following property: with (very) high probability their 
micro states are concentrated in an unusually small region of phase or configuration space. This allows us to speak of typical states and employ this concept instead of dealing with the full ensemble of micro states in a phase cell.

The basis of these results are the Levy-like inequalities and the Levy concentration theorems. A typical example is the law of large numbers and in our context the random graph model as it was used in [30]. The crucial point in all these examples is not that mean value and variance of certain random functions over some measure space do exist but that the variance is unusually small. This implies that a random function can be replaced probabilistically by its average, and configurations by the particular configurations belonging to the respective mean values.

\section{GRAPHS AS METRIC SPACES}

In this section we gather a few definitions and properties of graphs for completeness and show that they are metric spaces, so that all the results regarding metric spaces in this paper applies to them.

A graph, $G(V, E)$, consists of a set of points $V=\left\{v_{i}\right\}$ that are its vertices and a set of edges $E=\left\{e_{i j}\right\} \subset V \times V$ that connects some or all of the vertices. If $e_{i j}=e_{j i}$ the graph is called undirected otherwise it is directed. The number of edges incident on a vertex $v_{i}$ is called the degree or valency of that edge, $\operatorname{deg}\left(v_{i}\right)$. If each vertex of the graph has the same degree $k$ the graph is called a $k$-regular graph and the graph itself is said to have vertex degree $k$. If $\operatorname{deg}\left(v_{i}\right) \leq A$ for all $v_{i} \in V(G)$, then the graph has globally bounded vertex degree. The degree is called locally bounded if $\forall v_{i} \in V(G) \Rightarrow \operatorname{deg}\left(v_{i}\right) \in \mathbb{N}_{0}$. A graph is connected if every pair of vertices are connected by a finite edge sequence.

A path $\gamma$ is an edge sequence without repetition of vertices with the possible exception of initial and final vertex. The length $l(\gamma)$ of a path $\gamma$ is the number of edges occurring in the path. A geodesic path between two vertices is a path of minimum length.

One can define a natural metric on a graph by defining the distance between two vertices $v_{i}, v_{j}$ as the length of the geodesic path between them

$$
d_{G}\left(v_{i}, v_{j}\right):=\min _{\gamma}\left\{l(\gamma), \gamma \text { between } v_{i} \text { and } v_{j}\right\} .
$$

Then the graph $G$ together with this distance function is a metric space $\left(G, d_{G}\right)$.

Using this one can define a closed ball of radius $r$ centered around $v_{i}$ in a graph as

$$
B\left(v_{i}, r\right)=\left\{v_{j} \mid d\left(v_{i}, v_{j}\right) \leq r\right\},
$$

just like the definition in any metric space. Also the boundary of a ball is defined as

$$
\partial B\left(v_{i}, r\right)=\left\{v_{j} \mid d\left(v_{i}, v_{j}\right)=r\right\} .
$$

An open ball is defined as $B\left(v_{i}, r\right)-\partial B\left(v_{i}, r\right)$. One then defines the number of nodes in a ball and in the boundary of a ball as $\left|B\left(v_{i}, r\right)\right|,\left|\partial B\left(v_{i}, r\right)\right|$ respectively.

The growth function $\beta\left(G, v_{i}, r\right)$ starting from a vertex $v_{i}$ as a function of distance $r$ from it, is defined as

$$
\beta\left(G, v_{i}, r\right)=\left|B\left(v_{i}, r\right)\right| .
$$


Correspondingly we define

$$
\partial \beta\left(G, v_{i}, r\right)=\beta\left(G, v_{i}, r\right)-\beta\left(G, v_{i}, r-1\right)
$$

which is the difference between the number of nodes in a ball of radius $r$ and another one of radius $r-1$ both centered at $v_{i}$.

The growth function of a graph can have different forms, however, an important case for our purposes is when

$$
\beta\left(G, v_{i}, r\right) \lesssim r^{\alpha}, \quad \alpha \geq 0
$$

In that case the graph $G$ is said to have polynomial growth. For such a graph one can define the degree of polynomial growth as

$$
\bar{D}\left(G, v_{i}\right)=\limsup _{r \rightarrow \infty} \frac{\log \beta\left(G, v_{i}, r\right)}{\log r} .
$$

If $G$ has locally bounded vertex degree, and if $\exists A, B, \alpha$ such that

$$
A r^{\alpha} \leq \beta\left(G, v_{i}, r\right) \leq B r^{\alpha},
$$

for all $r>r_{0}$ for some $r_{0}$ and for all $A, B$ independent of the reference point $v_{i}$, then we say $G$ has uniform polynomial growth. The polynomial growth and its uniformness plays an important role both in convergence of the coarse graining process and in obtaining an integer dimension for spaces under considerations, as we will see later.

Remark IV.1. For more details see e.g. 24] and the literature cited there. We developed such notions already in [25] on physical grounds without being aware that a similar concept was used in geometric group theory ([43]).

Lemma IV.2. For locally bounded vertex degree the exponent $\bar{D}$ is independent of the vertex $v_{i}$, i.e. is a graph characteristic (see [25]).

\section{A GENERIC COARSE GRAINING SCHEME}

As mentioned in the previous sections, we are going to work in a sufficiently large class of metric spaces which we will call $\mathcal{S}$. We would like to introduce a coarse graining process $\mathcal{K}$ in $\mathcal{S}$ such that

$$
\begin{aligned}
\mathcal{K}: \quad \mathcal{S} \rightarrow \quad & \mathcal{S} \\
\left(M_{1}, d_{1}\right) & \mapsto\left(M_{2}, d_{2}\right), \quad\left(M_{1}, d_{1}\right),\left(M_{2}, d_{2}\right) \in \mathcal{S} .
\end{aligned}
$$

As we will explain below, we choose $\mathcal{K}$ to be a process that falls under the category of quasi-isometries. To understand what this means, we should start by introducing some preliminary concepts. The first concept is the notion of an isometric embedding. For brevity, we sometimes write a metric space $(M, d)$ as just $M$ when the context is clear.

Given two metric spaces $X, Y \in \mathcal{S}$, an isometric embedding $f$ of $X$ into $Y$

$$
f: X \rightarrow Y
$$

is a distance preserving map, i.e.

$$
d_{X}\left(x_{1}, x_{2}\right)=d_{Y}\left(f\left(x_{1}\right), f\left(x_{2}\right)\right), \quad \forall x_{1}, x_{2} \in X .
$$


It is an isometry if it is also surjective. A weaker but in our context much more useful and appropriate version is a quasi-isometric embedding. A map $f: X \rightarrow Y$ between two metric spaces is called a quasi-isometric embedding if $\exists \lambda \geq 1, \epsilon \geq 0$ such that

$$
\frac{1}{\lambda} d_{X}\left(x_{1}, x_{2}\right)-\epsilon \leq d_{Y}\left(f\left(x_{1}\right), f\left(x_{2}\right)\right) \leq \lambda d_{X}\left(x_{1}, x_{2}\right)+\epsilon, \quad \forall x_{1}, x_{2} \in X
$$

i.e. $\exists \lambda \geq 1, \epsilon \geq 0$ such that $\forall x_{1}, x_{2} \in X$, the distance between their images under $f$, is within a factor $\lambda$ and up to an additive constant of their original distances.

A quasi-isometric embedding $f: X \rightarrow Y$ is called a quasi-isometry if every point $y \in Y$ lies within a constant distance $C \geq 0$ of an image point,

$$
\forall y \in Y: \exists x \in X: d_{Y}(y, f(x)) \leq C .
$$

Quasi-isometry allows us to compare metric spaces neglecting their small-scale structure, i.e. it is an equivalence relation on metric spaces ignoring small-scale structure and just looking at the coarse structures (see example below). Note that here the important property is the transitivity, i.e., if $X, Y$ and $Y, Z$ are quasi-isometric then $X, Z$ are also quasi-isometric. In this context one should perhaps mention the approach of [26] on random metric spaces. If in the above definition, $\lambda=1$, then the map $f$ is called a rough isometry.

Remark V.1. In the following we will usually choose for convenience $C=\epsilon$ and call it an $\epsilon$-rough isometry. This is useful as we frequently study cases where both $\epsilon, C$ approach zero.

Quasi-isometry can actually be restated in a symmetric way. Two metric spaces $X$ and $Y$ are quasi-isometric if there exists $\lambda \geq 1, \epsilon \geq 0, \rho \geq 0$ and maps

$$
f: X \rightarrow Y, \quad g: Y \rightarrow X
$$

such that for all $x, x_{1}, x_{2} \in X$ and $y, y_{1}, y_{2} \in Y$,

$$
d_{Y}\left(f\left(x_{1}\right), f\left(x_{2}\right)\right) \leq \lambda d_{X}\left(x_{1}, x_{2}\right)+\epsilon, \quad d_{X}\left(g\left(y_{1}\right), g\left(y_{2}\right)\right) \leq \lambda d_{Y}\left(y_{1}, y_{2}\right)+\epsilon
$$

and

$$
d_{X}(g \circ f(x), x) \leq \rho, \quad d_{Y}(f \circ g(y), y) \leq \rho .
$$

Such a map $g$ is called a quasi-inverse (see e.g. [24] or [43, 44])

As a simple but interesting example of quasi-isometry, we note that the integer lattice $\mathbb{Z}^{n}$ is quasi-isometric to $\mathbb{R}^{n}$. This can be shown by considering the quasi-isometry map

$$
\begin{aligned}
f & : \mathbb{Z}^{n} \rightarrow \mathbb{R}^{n} \\
& :\left(x_{1}, \ldots, x_{n}\right) \mapsto\left(x_{1}, \ldots, x_{n}\right), \quad x_{i} \in \mathbb{Z} .
\end{aligned}
$$

The map is metric-preserving if we use the Euclidean metric in both spaces. It is perhaps more natural if we employ the intrinsic graph metric (defined below) on $\mathbb{Z}^{n}$, that is, the minimal number of steps on the lattice $\mathbb{Z}^{n}$. In that case we have a true quasi-isometry. In the former case we have $\lambda=1$ and $\epsilon=0$, while $n$-tuples $\in \mathbb{R}^{n}$ are within a distance $C=\sqrt{\frac{n}{4}}$ of $n$-tuples $\in \mathbb{Z}^{n}$ with respect to the usual Euclidean metric. On the other hand, the map

$$
\begin{aligned}
g & : \mathbb{R}^{n} \rightarrow \mathbb{Z}^{n} \\
& :\left(y_{1}, \ldots, y_{n}\right) \mapsto\left(x_{1}, \ldots, x_{n}\right), \quad y_{i} \in \mathbb{R}
\end{aligned}
$$


rounding $n$-tuples $\in \mathbb{R}^{n}$ to the nearest $n$-tuple $\in \mathbb{Z}^{n}$ is also a quasi-isometry. In this case, the distance between pairs of points is changed by adding or subtracting at most $2 \sqrt{\frac{n}{4}}$. Also we can see that

$$
d_{X}(g \circ f(x), x)=0
$$

and

$$
d_{Y}(f \circ g(y), y) \leq \sqrt{\frac{n}{4}}
$$

which means that for this example, $\rho=\sqrt{\frac{n}{4}}$.

Now we are in a position to make our definition of coarse graining more precise. In our approach, a coarse graining map $\mathcal{K}$ is a quasi-isometry $\mathcal{K}: X \rightarrow Y$, while not all quasi-isometries can be regarded as coarse grainings. The particular form of such a coarse graining has of course to be motivated by the physical context. It implies that details or finer DoF are deleted or summed over and that we go over to coarser substructures in each step. Therefore not every quasi-isometry will do.

Using a coarse graining operation $\mathcal{K}$, we can generate a sequence of metric spaces, $\left\{\left(M_{i}, d_{i}\right)\right\}, i=0, \ldots, N$, such that,

$$
\left(M_{0}, d_{0}\right) \stackrel{\mathcal{K}}{\longrightarrow}\left(M_{1}, d_{1}\right) \stackrel{\mathcal{K}}{\longrightarrow}\left(M_{2}, d_{2}\right) \stackrel{\mathcal{K}}{\longrightarrow} \cdots \stackrel{\mathcal{K}}{\longrightarrow}\left(M_{N}, d_{N}\right)
$$

where all $\left(M_{i}, d_{i}\right) \in \mathcal{S}$, and $\left(M_{i+1}, d_{i+1}\right)$ is a coarse grained version of $\left(M_{i}, d_{i}\right)$ in this sequence. We also write

$$
\left(M_{i+j}, d_{i+j}\right)=\underbrace{\mathcal{K}(\mathcal{K}(\cdots \mathcal{K}}_{j \text { times }}\left(\left(M_{i}, d_{i}\right)\right)))=\mathcal{K}^{j}\left(\left(M_{i}, d_{i}\right)\right) .
$$

Then the main idea is that after sufficiently many steps of such coarse grainings, one will hopefully arrive at a final limit space that is stable under further coarse graining $\mathcal{K}$, similar to a fixed point in the renormalization group analysis2. Thus we expect this "fixed point" (see below), if it does exist, to exhibit certain selfsimilarity properties as was studied in [20]. Furthermore, as in some of the renormalization group construction on, say, a lattice, all the intermediate spaces remain discrete, and it is only after a rescaling procedure that the fixed point is expected to behave as a smooth structure, resembling our macroscopic space-time.

One should say that a fixed point in the strict sense may not exist. We rather expect that we have what we call a macroscopic fixed point which comprises an ensemble of microstates looking the same macroscopically. This macroscopic fixed point is expected to be stable under further coarse graining transformations while the microstates move in the class belonging to the macroscopic fixed point.

\section{A. Some concrete examples of coarse graining $\mathcal{K}$}

It is illustrative and important to describe various graph transformations which are physically relevant to coarse graining and belong to the class of quasi-isometries or rough isometries. Two of such operations, edge insertions and edge deletions, we presented in observation 2.7 of [24]. There we employed these processes in the context of a notion of

${ }^{2}$ Such a program was motivated in e.g. 32. 
dimension of networks/graphs. One can easily see that the respective proofs, which can be found in [32] and [25], apply also in our case of quasi-isometry 3. Below we will present a few more of such transformations that constitute coarse graining in the sense that they represent a transition $G \rightarrow G^{\prime}$ in which some of the finer degrees of freedom in $G$ are ignored or averaged over.

\section{Quasi-isometry coarse graining}

\section{$k$-local edge insertion/deletion}

A $k$-local insertion/deletion of edges is the insertion/deletion of arbitrarily many edges in the $k$-neighborhood of vertices of a locally finite graph $G$. In the case of edge deletions the procedure is slightly more involved, that is, it refers rather to $k$-neighborhoods in the new graph $G^{\prime}$. I.e., edges are deleted between vertices which have a distance smaller than $k$ in $G^{\prime}$. It is tacitly assumed that $G^{\prime}$ is still connected. Note furthermore that the maximal number of possible edge insertions in a $k$-neighborhood of a vertex is bounded in a locally finite graph. The resulting graph $G^{\prime}$ is quasi-isometric to $G$ (for more details see Observation 2.7 in [24]).

\section{Vertex contraction of diameter $\leq k$}

Another graph transformation $G \rightarrow G^{\prime}$ consists of the following steps. In $G$, take the subgraphs $H_{i}^{G_{k}}$ of diameter $\leq k$ and contract them to a single vertex. These will be the vertices $v_{i}^{\prime} \in G^{\prime}$. An edge $e_{i j}^{\prime} \in E\left(G^{\prime}\right)$, pointing from $v_{i}^{\prime} \in G^{\prime}$ to $v_{j}^{\prime} \in G^{\prime}$ is drawn in $G^{\prime}$, if there are edges in $G$ that point from $H_{i}^{G_{k}}$ to $H_{j}^{G_{k}}$. In case several edges in $G$ point from $H_{i}^{G_{k}}$ to $H_{j}^{G_{k}}$, we have two options. Either they are replaced in $G^{\prime}$ by a single edge, or they can inhere an edge color based on the number (and/or color) of edges in $G$ pointing from $H_{i}^{G_{k}}$ to $H_{j}^{G_{k}}$, that are identified as the edge $e_{i j}^{\prime} \in G^{\prime}$. The latter is an interesting options which makes it possible that the coarse grained graph $G^{\prime}$ can have different (and coarse grained) edge colors that are inherited from the underlying graph $G$. This may have interesting and possibly deep consequences in theories like loop quantum gravity and group field theory where the color of edges represent the irreducible representation of the local gauge group of the macroscopic theory.

Conclusion V.2. All these $k$-local graph transformations lead to a quasi-isometric new graph $G^{\prime}$ and in general they are true quasi-isometries (i.e., not rough isometries), in particular if they are performed globally on the infinite graph $G$.

\section{Rough isometry coarse graining}

\section{Clique graph transformation $\mathcal{C}(G)$}

This is the transition from a graph $G$ to its so called clique graph $\mathcal{C}(G)$. A Cliques or a maximals subsimplex in a graph $G$ is a subset of $G$ in which all the vertices are connected

\footnotetext{
3 At the time of writing [25, 32] we were not aware of the various mathematical notions we used in [24].
} 
with each other. Cliques and clique graphs play an important role in graph theory (cf. e.g. [45] or [46, 47]) and we used them already in [30, 32]. Our motivation was mainly physical as we tried to find a substitute for the block spins of the ordinary Wilsonian renormalization group in strongly erratic and disordered systems.

The clique graph $\mathcal{C}(G)$ of $G$ is defined as a graph whose vertices are the cliques of $G$, and an edge exists between two of vertices of $\mathcal{C}(G)$ if the underlying cliques have non-zero vertex overlap (in $G$ ). Notice that in this case, the number of these vertices in $G$ that are shared between two cliques, can give rise to colors of edges in $\mathcal{C}(G)$ in different manners, hence leading to emergent edge colors in $\mathcal{C}(G)$. However, in this case, these emergent colors do not seem to have anything to do with edge colors in $G$, but rather the information related to the vertices of $G$. Now we present an important theorem.

Theorem V.3. If $G$ has globally bounded vertex degree deg $(G)$, its clique graph $\mathcal{C}(G)$ has also globally bounded vertex degree, and is roughly isometric to $G$ with $C=\epsilon=1$.

See Appendix B for a proof.

\section{THE GROMOV-HAUSDORFF SPACE OF METRIC SPACES}

Having defined a coarse graining procedure $\mathcal{K}$ which generates a coarse grained sequence $\left\{\left(M_{i}, d_{i}\right)\right\}_{i=0, \ldots, N}^{\mathcal{K}}$, it is possible to introduce some kind of a distance function $d_{\mathcal{S}}$ between suitable metric spaces, with respect to which one can ask if the sequence converges to a limit space, and to what extent two spaces are structurally similar or different.

We begin with the simpler notion of the distance of two subsets of a metric space. Given a metric space $\left(Z, d_{Z}\right)$ and two of its nonempty subsets $X, Y \subset Z$, one can define a distance between these subsets called the Hausdorff distance $d_{H}^{Z}$ as

$$
d_{H}^{Z}(X, Y)=\max \left\{\sup _{x \in X} \inf _{y \in Y} d_{Z}(x, y), \sup _{y \in Y} \inf _{x \in X} d_{Z}(x, y)\right\}
$$

or

$$
d_{H}^{Z}(X, Y)=\inf \left\{\epsilon \geq 0 \mid X \subseteq U_{\epsilon}(Y), Y \subseteq U_{\epsilon}(X)\right\} .
$$

Here, $U_{\epsilon}(X)$ is the $\epsilon$-neighborhood of a subset $X \subset Z$ of a metric space $\left(Z, d_{Z}\right)$, and it is defined as

$$
U_{\epsilon}(X)=\bigcup_{x \in X}\{z \in Z \mid d(z, x) \leq \epsilon\},
$$

i.e., it is the union of all $\epsilon$-balls around all $x \in X$, or the set of all points in $Z$ that are within a distance $\epsilon$ of the set $X$, or the generalized ball of radius $\epsilon$ around $X$.

The Hausdorff distance makes the set of non-empty compact subsets of a complete metric space into a complete metric space [44, 48]. Note that on the set of all non-empty (not necessarily compact) subsets of $Z$, in general $d_{H}^{Z}$ only defines a pseudometric, viz., with $A, B \subset Z$, then $d_{H}^{Z}(A, B)=0$ does not necessarily mean $A \neq B$.

With the notions of the Hausdorff distance and isometric embedding, Gromov [42, 44, 49] was able to develop a distance concept between two arbitrary compact metric spaces. This distance is called the Gromov-Hausdorff distance $d_{G H}$ between two compact metric spaces $X, Y$ and is defined as

$$
d_{G H}(X, Y)=\inf d_{H}^{Z}(f(X), g(Y))
$$


for all metric spaces $Z$ and all isometric embeddings $f: X \rightarrow Z$ and $g: Y \rightarrow Z$. Equivalently it is defined as

$$
d_{G H}(X, Y)=\inf d_{H}^{X \sqcup Y}(f(X), g(Y)),
$$

where $X \sqcup Y$ is the disjoint union of $X, Y$, and the metric $d_{H}^{X \sqcup Y}$ extending the metrics on $f(X), g(Y)$. This latter version was particularly used in [49].

This distance has some interesting properties:

1. On the set of all isometry classes of compact metric spaces, it provides a metrid 4 . This set together with $d_{G H}$ is a complete metric space called the Gromov-Hausdorff space [49].

2. In general, however, it provides only a pseudo-metric. This holds for example if $X$ is dense in $Y$, or if $X, Y$ are isometric.

3. It measures how far two compact metric spaces are from being isometric, i.e. $X, Y$ are isometric iff $d_{G H}(X, Y)=0$. It is is in fact a measure of metric similarity.

4. It defines a notion of convergence for sequences of compact metric spaces, called the Gromov-Hausdorff convergence.

In the Gromov-Hausdorff space, a metric space to which a sequence of compact metric spaces converges in $d_{G H}$ is called its Gromov-Hausdorff limit5.

We now see that given these notions, one can (in principle) check if in the GromovHausdorff space, a sequence of metric spaces, generated by a concept of coarse graining $\mathcal{K}$, or scale transformation (see below), has a Gromov-Hausdorff limit.

However, many of the spaces that are interesting physically are non-compact and hence do not belong to the Gromov-Hausdorff space. Thus we need to somehow extend these notions to sequences of coarse grained spaces, $\left\{\left(M_{i}, d_{i}\right)\right\}_{i=0, \ldots, N}^{\mathcal{K}}$, that are non-compact, at least to a relevant subset of these non-compact metric spaces. This was also done by Gromov. More precisely, he extended this notion of convergence in $d_{G H}$, to non-compact but locally compact metric spaces $\mathcal{S}$. To see this, we need the concept of pointed metric spaces:

Definition VI.1. A locally compact complete metric space $\left(X_{i}, d_{i}\right)$ with a distinguished point $x_{i} \in X_{i}$, is called a pointed metric space. It is denoted by $\left(\left(X_{i}, d_{i}\right), x_{i} \in X_{i}\right)$.

The extension of the Gromov-Hausdorff convergence to $\mathcal{S}$ is then done in the following way:

Definition VI.2. A sequence of pointed metric spaces $\left\{\left(\left(X_{i}, d_{i}\right), x_{i} \in X_{i}\right)\right\}$ is defined to converge to $\left(\left(X, d_{X}\right), x \in X\right)$, if for all $r>0$, the sequence of closed $r$-balls, $\left\{B\left(x_{i}, r\right) \subset X_{i}\right\}$, converges to $B(x, r) \subset X$ in $d_{G H}$. This is called pointed $\boldsymbol{G H}$-convergence.

Thus in effect, if we have a sequence of non-compact but locally compact metric spaces, we are still able to draw a conclusion if they converge to a locally compact space in pointed GH-sense. We take this space of non-compact but locally compact metric spaces as our super space $\mathcal{S}$ with which we will work. It is quite similar in concept, to the "theory space" of the renormalization methods. As we will see in section VIII, this extension of GromovHausdorff convergence to this superspace plays a crucial role in our enterprise. It is worth

\footnotetext{
4 cf. lemma 4.6 in 24 ].

${ }^{5}$ For brevity we may use GH instead of Gromov-Hausdorff in some parts of the text.
} 
mentioning that two very interesting types of non-compact spaces that are locally compact are $\mathbb{R}^{n}$, and consequently finite dimensional non-compact topological manifolds, since they share the local properties of the Euclidean spaces 6 .

The concepts of GH-convergence or GH-distance seem to be quite abstract compared to the simpler concept of the Hausdorff-distance, but note that it yields much more detailed information about the structure of the spaces under discussion. Hausdorff-distance simply measures the metrical distance of sets as subsets of a larger metric space. However, the GHdistance, due to the incorporation of all admissible Hausdorff distances, actually measures the structural relatedness (or similarity) of spaces. This is much more specific, and in line with the general notion of coarse graining in physics.

Due to computational complications, it is seldom possible to calculate the exact GHdistance between two metric spaces?. However, it is usually possible and sufficient to obtain efficient upper bounds. In this respect the second version of GH-distance, equation (6.2), is quite useful 8 .

To get a better feeling how quasi-isometry or rough isometry and GH-distance are structurally related we present an important theorem 9 that has far reaching consequences and plays an important role in discussions about the convergence.

Theorem VI.3. Two metric spaces $X, Y$, have finite GH-distance iff they are roughly isometric (i.e., with the $\lambda=1$ and $C=\epsilon$ in (5.5) and (5.6)). Furthermore it holds in particular:

$$
\frac{1}{2} d_{G H}(X, Y) \leq \inf \{\epsilon\} \leq 2 d_{G H}(X, Y)
$$

with the $\epsilon$ 's belonging to $\epsilon$-rough isometries between $X, Y$.

The proof along with a brief discussion can be found in [24].

An implication of this theorem is that since a finite $\mathrm{GH}$-distance between $X, Y$, is equivalent to $X, Y$ being $\epsilon$-roughly isometric,

$$
\left|d_{Y}\left(f(x), f\left(x^{\prime}\right)\right)-d_{X}\left(x, x^{\prime}\right)\right| \leq \epsilon,
$$

then quasi-isometric spaces, when the quasi-isometry is not a rough isometry, have an infinite $\mathrm{GH}$-distance. That means if $\mathcal{K}$ is such an operation performed on space $G$, then $d_{G H}(G, \mathcal{K}(G))=\infty$ and thus $G$ and $\mathcal{K}(G)$ are structurally quite different metric-wise. They are in fact infinitely apart from being isometric (see item 3 above). Another observation is that, a sequence

$$
\{G, \mathcal{K}(G), \mathcal{K}(\mathcal{K}(G)), \ldots\}
$$

for which all the steps of coarse graining are true quasi-isometries cannot converge in $d_{G H}$. We will discuss this point in more detail in the following sections. Note that a point in the coarse graining sequence where true quasi-isometry changes to rough isometry may be called a geometric phase transition point as we recognize a transition from structural dissimilar to structural similar spaces.

Another illuminating observation is the following. Assume that we have a sequence of metric spaces, $\left\{X_{\nu}\right\}$, which converges in $d_{G H}$ towards some metric space $X$. Then by the

${ }^{6} \mathbb{R}^{n}$ is locally compact due to the Heine-Borel theorem

7 This is a consequence of the need to incorporating all admissible metrics (Hausdorff distances), which ironically makes the $d_{G H}$ notion so powerful.

8 To get more acquainted with the technical subtleties see section 4 of [24]. The crucial point is always the verification of the triangle inequality.

9 This is the theorem 4.15 in [24]. It is also contained in the form given here in 50] 
same token, there exists a sequence of rough $\epsilon_{\nu}$-isometries, $f_{\nu}$, between $X_{\nu}$ and $X$ with $\epsilon_{\nu} \rightarrow 0$. This implies (by definition) that

$$
\lim _{\epsilon_{\nu} \rightarrow 0} f_{\nu}\left(X_{\nu}\right) \text { is dense in } X
$$

with

$$
\lim _{\epsilon_{\nu} \rightarrow 0} d_{X}\left(f_{\nu}\left(x_{\nu}\right), f_{\nu}\left(x_{\nu}^{\prime}\right)\right)=\lim _{\epsilon_{\nu} \rightarrow 0} d_{X_{\nu}}\left(x_{\nu}, x_{\nu}^{\prime}\right) .
$$

Thus we say that in the limit $\epsilon_{\nu} \rightarrow 0$, the spaces $X_{\nu}, X$ become essentially isometric, in order to have a label for this asymptotic behavior.

\section{CONVERGENCE AND CONTINUUM LIMIT I}

The deep question of under what physical and/or mathematical conditions a sequence of coarse-grained spaces has a macroscopic or continuum limit, will be postponed to the next section. In this section we will discuss various topics related to the rescaling of our metric spaces, and the fixed point in this rescaling process (some results can also be found in $[24,50])$.

Since we will be using some concepts from dynamical systems, let us begin by some related definitions. In a dynamical system with a space of admissible states (or phase space) $H$, an attractor or an attracting set is, roughly speaking, a closed subset $A \subset H$ such that for many choices of initial states $I_{A} \subset H$, the system will eventually evolve to $A$. The set of initial conditions $I_{A}$ for which the system's state eventually evolves to $A$ is called the basin of attraction of $A$.

Now we explore the relation of the above notions with our framework. If we have a metric space, $X$, and a metric $d_{X}$ on it, we can define, in a canonical way, a whole sequence of scaled metrics $\lambda \cdot d_{X}$ with $\lambda \in \mathbb{R}^{+}$. The limit $\lambda \rightarrow 0$ corresponds to the large scale structure of $X$, while $\lambda \rightarrow \infty$ reveals the fine structure of $X$ by magnifying the infinitesimal neighborhoods of the points of $X$. In our context the limit $\lambda \rightarrow 0$ is of particular importance.

We assume that the GH-limit

$$
\left(X_{\infty}, d_{\infty}\right):=\lim _{\lambda \rightarrow 0}\left(X, \lambda d_{X}\right)
$$

exists with metric $d_{\infty}=\lim _{\lambda \rightarrow 0} \lambda d_{X}$, and we want to infer some general properties of this limit space.

Observation VII.1. From what we have learned in the last section, all spaces, $\left\{\left(X^{\prime}, d_{X^{\prime}}\right) \mid d_{G H}\left(X^{\prime}, X\right)<\infty\right\}$ have the same limit $\left(X_{\infty}, d_{\infty}\right)$. Furthermore, it is easy to see that $\left(X_{\infty}, d_{\infty}\right)$ is the only scaling limit in this set.

This set of spaces, $\left\{\left(X^{\prime}, d_{X^{\prime}}\right)\right\}$ (including $\left(X, d_{X}\right)$ itself), is the basin of attraction of the attractor $\left(X_{\infty}, d_{\infty}\right)$ under the evolution map

$$
\phi_{\lambda}:\left(X^{\prime}, d_{X^{\prime}}\right) \longmapsto\left(X^{\prime}, \lambda d_{X^{\prime}}\right)
$$

for $\lambda \rightarrow 0$. This limit space $\left(X_{\infty}, d_{\infty}\right)$ has the following nice property: 
Observation VII.2. $\left(X_{\infty}, d_{\infty}\right)$ is scale invariant under every scaling map

$$
\phi_{l}:\left(X_{\infty}, d_{\infty}\right) \longmapsto\left(X_{\infty}, l d_{\infty}\right)
$$

in the sense that

$$
d_{G H}\left(X_{\infty}, l X_{\infty}\right)=0 .
$$

This implies that there exists an essentially isometric map for every $\phi_{l}$ and, as a consequence, a scaling map, $f_{l}$, from $X_{\infty} \rightarrow X_{\infty}$, i.e., we have

$$
d_{\infty}\left(x, x^{\prime}\right)=l \cdot d_{\infty}\left(f_{l}(x), f_{l}\left(x^{\prime}\right)\right) .
$$

Proof. With $\lim _{\lambda \rightarrow 0}\left(X, \lambda d_{X}\right)=\left(X_{\infty}, d_{\infty}\right)$, it holds that $\lim _{l \lambda \rightarrow 0}\left(X, l \lambda d_{X}\right)=\left(X_{\infty}, d_{\infty}\right)$ in GHsense. On the other hand, we have $\lim _{l \lambda \rightarrow 0}\left(X, l \lambda d_{X}\right)=l \cdot \lim _{\lambda \rightarrow 0}\left(X, \lambda d_{X}\right)=l \cdot\left(X_{\infty}, d_{\infty}\right)$.

We now see the following:

Conclusion VII.3. One may call $\left(X_{\infty}, d_{\infty}\right)$ a fixed point of the scaling map $\phi_{\lambda}$ for $\lambda \rightarrow 0$ in its basin of attraction given by $\left\{\left(X^{\prime}, d_{X^{\prime}}\right) \mid d_{G H}\left(X^{\prime}, X\right)<\infty\right\}$.

Some examples of scale invariant spaces are e.g. $\mathbb{R}^{n}$ or various fractal spaces.

\section{CONVERGENCE AND CONTINUUM LIMIT II}

In this section we want to develop criteria under which a sequence of metric spaces has a limit space under $\mathcal{K}$. Crucial in this respect is the Gromov-compactness theorem. As this argument is quite intricate and was already discussed in [24] we will only briefly recapitulate the relevant points for the sake of completeness and will, refer the reader to [24] for more details.

A family of compact spaces, $X_{\lambda}$, is called uniformly compact if their diameters are uniformly bounded and if for each $\epsilon>0, X_{\lambda}$ is coverable by $N_{\epsilon}<\infty$ balls of radius $\epsilon$ independent of the index $\lambda$. We then have the fundamental result, derived by Gromov [51]:

Theorem VIII.1. A sequence of metric spaces $\left\{\left(X_{i}, d_{i}\right)\right\}$ contains a convergent subsequence in $d_{G H}$, iff it is uniformly compact.

The proofs typically use an Arzela-Ascoli-Cantor-diagonal-sequence-like argument 44, 49, 51]. This theorem can immediately be extended to the sequences of pointed metric spaces in $\mathcal{S}$.

Theorem VIII.2. If for all $r$ and $\epsilon>0$ the balls $B\left(x_{i}, r\right)$ of a given sequence of proper metric spaces $\left\{\left(X_{i}, x_{i} \in X_{i}\right)\right\}$ are uniformly compact, then a subsequence of spaces converges in pointed GH-sense.

Note that this means that the balls converge in the usual GH-sense. But the convergence is not uniform.

It is perhaps helpful to illustrate these results by giving a simple example. Take the lattice $\mathbb{Z}^{n}$ embedded in $\mathbb{R}^{n}$ and take the scaling limit

$$
\phi_{l}:\left(\mathbb{Z}^{n}, d_{\mathbb{Z}^{n}}\right) \longmapsto\left(\mathbb{Z}^{n}, \lambda d_{\mathbb{Z}^{n}}\right), \quad \lambda=2^{-l}
$$


where $d_{\mathbb{Z}^{n}}$ is a suitable metric on $\mathbb{Z}^{n}$ (see below). For $\lambda \rightarrow 0$, i.e. $l \rightarrow \infty$, we have

$$
\lim _{\lambda \rightarrow 0}\left(\mathbb{Z}^{n}, \lambda d_{\mathbb{Z}^{n}}\right)=\left(\mathbb{R}^{n}, d_{\mathbb{R}^{n}}\right),
$$

which holds only in pointed GH-sense since the convergence is not uniform. Here the metric of the scaling limit, $d_{\mathbb{R}^{n}}$, depends on $d_{\mathbb{Z}^{n}}$. For example if we use the Euclidean metric on $\mathbb{Z}^{n}$, the limit space $\mathbb{R}^{n}$ also carries the ordinary Euclidean metric. But if we use the graph metric or taxicab metric on $\mathbb{Z}^{n}$, the limit metric is also the taxicab metric (or $l^{1}$-metric) on $\mathbb{R}^{n}$. In this example, for a fixed ball around $x=0$ we can infer from what we said in theorem VI.3 that for $l \rightarrow \infty$ the ball is more and more filled with points stemming from lattices having edge length $2^{-l}$. It is in this way that we can envisage the pointed GH-convergence in the scaling situation.

To use this theorem effectively, we need practical and easy to control properties, that imply that a sequence of spaces is uniformly compact. We will supply properties which hold in particular in the situation we are interested in, that is, (infinite) networks/graphs. We begin with versions of the doubling property. A metric space is called doubling if each ball, $B\left(x_{i}, r\right)$, can be covered by at most $C$ balls with radius half that of $B\left(x_{i}, r\right)$, with $C$ independent of the balls $B$. It easily follows via iteration that this implies that $B$ is coverable by $C^{k}$ balls of radius $2^{-k}$.

There is a nice relation of this property to a more manageable case as follows: With $(X, d)$ a metric space, and $\mu$ a positive Borel measure on $X$, then $\mu$ is said to be doubling, if there exists a positive constant, $C$, being independent of $B$ such that

$$
\mu(2 B) \leq C \cdot \mu(B)
$$

for all balls in $X$. Here $2 B$ is a ball with the same center as $B$ but twice the radius. Then it follows that

$$
\mu\left(2^{k} B\right) \leq C^{k} \cdot \mu(B)
$$

We then have the theorem

Theorem VIII.3. If $(X, d)$ has a doubling measure, it is doubling as a metric space.

For a proof see [42], p.412 (the chapter being written by Semmes).

Now, defining a sequence of spaces $\left\{\left(X_{n}, d_{n}\right)\right\}$ to be uniformly doubling if the above doubling properties hold uniformly in it, we have

Theorem VIII.4. A sequence of spaces is uniformly compact if it is uniformly doubling.

It turns out that the latter property is more manageable than the former one: Consider a graph $(G, d)$ of uniform polynomial growth, for which

$$
A r^{d} \leq \beta(x, r) \leq B r^{d}
$$

for all $r \geq r_{0}$ and $A, B$ independent of the reference point $x$. Taking the sequence of scaled graphs $\left\{\left(G_{n}, d_{n}=n^{-1} \cdot d\right)\right\}$ made from $G$, we can prove $([24])$

Conclusion VIII.5. A graph with uniform polynomial growth has a doubling counting measure for sufficiently large $r \geq r_{0}$ and is hence doubling as a metric space for sufficiently large $r \geq r_{0}$. This implies that all balls $B_{n}(x, r)$ in $\left\{G_{n}, d_{n}\right\}$, where $G_{n}$ are of uniform polynomical growth, are uniformly compact. We conclude that there exists a subsequence of $\left\{\left(G_{n}, d_{n}=n^{-1} \cdot d\right)\right\}$, that converges in pointed $G H$-sense. 
This conclusion mean that the continuum limit, $\left(G_{\infty}, d_{\infty}\right)$, discussed in the preceding subsection, exists for graphs of uniform polynomial growth with rescaling map

$$
\phi_{\lambda}:(G, d) \longmapsto(G, \lambda d)=\left(G, n^{-1} d\right)=\left(G_{n}, d_{n}\right)
$$

where

$$
\left(G_{\infty}, d_{\infty}\right)=\lim _{\lambda \rightarrow 0} \phi_{\lambda}((G, d))=\lim _{\lambda \rightarrow 0}(G, \lambda d)
$$

\section{THE GEOMETRIC RENORMALIZATION GROUP IN THE SUPERSPACE OF METRIC SPACES}

We have now the necessary methods at our disposal in order to develop a geometric version of a Wilsonian renormalization group (RG) in our superspace of metric spaces. These consist of a general concept of coarse graining, a notion of continuum limit, and the idea of typicality of micro states lying in a phase cell describing some macro state like our continuum space time.

To begin with, we have argued in section III that, instead of dealing with possibly a complicated ensemble structure in our superspace $\mathcal{S}$, we can perform the coarse graining process on certain selected micro states, for example networks or graphs. This coarse graining process was then described in section $\mathrm{V}$, at least as far as its general characteristics are concerned. This process may may differ slightly from one metric space to another, depending on the type of the space, but the central pieces are an averaging and/or purification of certain substructures.

In the cases which interest us most, i.e. networks/graphs, this averaging consists typically of the replacement of particular subgraphs (like cliques), by vertices on the next coarse graining level, as described in section $\mathrm{V}$. The purification consists of adding/deleting edges or even whole subgraphs according to certain principles. In [32] the substructures were cliques and we deleted cliques which were unusually small, or edges in the clique graph if the overlap of the respective cliques was too marginal. The whole process tries to simulate the block spin approach of the ordinary real space Wilsonian renormalization group with the cliques in our example representing the blocks.

Observation IX.1. Note that the repetition of this coarse graining process does not leave the subregime of discrete graphs/networks. Thus we have to supplement it by a second type of process, a rescaling, as described in the two preceding sections. This then yields a continuum limit not only for the final limit space of a coarse graining process, but also for the various stages (or spaces) before that.

Furthermore, in contrast to the ordinary Wilsonian RG, which typically lives on simple Bravais lattices, the latter process of rescaling is also quite complicated to perform on highly irregular spaces.

Starting from some initial graph/network $G_{0}$, which we presume represents the pregeometry of our space-time on the most fundamental level, and neglecting the possible micro states carried by the vertices and edges 10 , we apply a sequence of coarse graining operations

${ }^{10}$ As mentioned before, in this first work, we take the simplest cases where the color of vertices and edges play no role in the coarse graining scenario. 
$\mathcal{K}$ on $G_{0}$, which then yields a coarse grained sequence of spaces $\left\{\left(G_{i}, d_{i}\right)\right\}, i=0, \ldots, N$, depicted as

$$
\left(G_{0}, d_{0}\right) \stackrel{\mathcal{K}}{\longrightarrow}\left(G_{1}, d_{1}\right) \stackrel{\mathcal{K}}{\longrightarrow}\left(G_{2}, d_{2}\right) \stackrel{\mathcal{K}}{\longrightarrow} \cdots \stackrel{\mathcal{K}}{\longrightarrow}\left(G_{n}, d_{n}\right)
$$

According to our assumptions, these coarse graining operators will belong to the class of quasi-isometries, including rough isometries.

Remark IX.2. As we will mention at the end of section $\underline{X}$, we possibly may have to deal with models of $S$ - $T$ which have both a near-and a far-order structure on a primordial scale, being generated by a sparse network of translocal edges. In that case it may happen that we have to leave the class of quasi-isometric coarse graining which was based on k-local operations.

We expect that, at least in the first steps, $\mathcal{K}$ will consist of a large number of subgraph contractions and edge deletions/insertions according to our fixed coarse graining protocol. Hence, in general, $\mathcal{K}$ will be a true quasi-isometry and not a rough isometry. As we saw above, in that case the GH-distance between consecutive graphs is infinite and thus they will differ structurally. On the other hand we observed in [32] that after a number of coarse graining steps the corresponding graphs had the tendency of becoming more regular and structurally more similar. This motivates us to formulate our central RG-conjecture:

Conjecture IX.3. If we start from suitable initial graphs which display a certain kind of (hidden) selfsimilarity, we expect that our coarse grained graphs will change their character after a number of coarse graining steps and become roughly isometric, i.e. structurally similar.

This means that after, say, step $m$ of the coarse graining, the operation

$$
\mathcal{K}:\left(G_{m-1}, d_{m-1}\right) \rightarrow\left(G_{m}, d_{m}\right)
$$

becomes a rough isometry. Note that operationally $\mathcal{K}$ obeys the same protocols (contract cliques to new vertices etc.), but due to the change of structure of our graphs, this operation now yields a space that is roughly isometric to the previous one.

At this point we should spell out a warning. In the introductory sections we introduced the idea of phase cells of microstates which make up the observable continuum limit spacetime manifold S-T. We learned previously that roughly isomorphic spaces have finite GHdistance and thus have the same continuum limit. On the other hand, we cannot expect that in the above sequence of coarse graining steps the roughly isometric spaces converge to a limit on the microscopic level. What we will observe however is that they belong to a joint macrocopic continuum limit space (i.e. our classical space-time). To be more precise we have the following.

Observation IX.4. In general a sequence of roughly isometric spaces, while being structurally similar, are not uniformly compact, thus there will not exist in the generic case a GH-convergent subsequence. This can for example be seen in the transition from a graph to its clique graph (cf. the numerical estimates in [32]). While the two spaces are roughly isometric the number of cliques may strongly increase so that the doubling property is not fulfilled. On the other hand we studied very simple and regular examples in section 4 of [32] and found real fixed points or accumulation points. That is, it may be that both cases may happen while we think, the latter case is not the generic one. 
Note in particular that on the discrete graph-level the GH-distance is discrete. This implies that the smallest possible distances are zero or one. This means that limit points on the discrete level, if they exist at all, are attained after a finite number of steps and remain stable under further coarse graining. The same happens with possible accumulation points. These cases are illustrated by the above mentioned examples.

In order to complete our coarse graining picture we now turn our attention to the continuum limit of these spaces. In a first step we present a theorem which turns out to be very useful in our context and which we proved in [24].

Theorem IX.5. Let $G_{1}, G_{2}$ both have globally bounded vertex degree, let $G_{1}$ have uniform polynomial growth, and let $G_{1}, G_{2}$ be quasi-isometric. Then also $G_{2}$ has uniform polynomial growth. Thus in a sequence of graphs $\left\{\left(G_{i}, d_{i}\right)\right\}, i=0, \ldots N$ each derived from the previous one by a quasi-isometric coarse graining process, each member has its own scaling limit as shown in theorem VIII.5.

This can be illustrated by means of the following graphical representation.

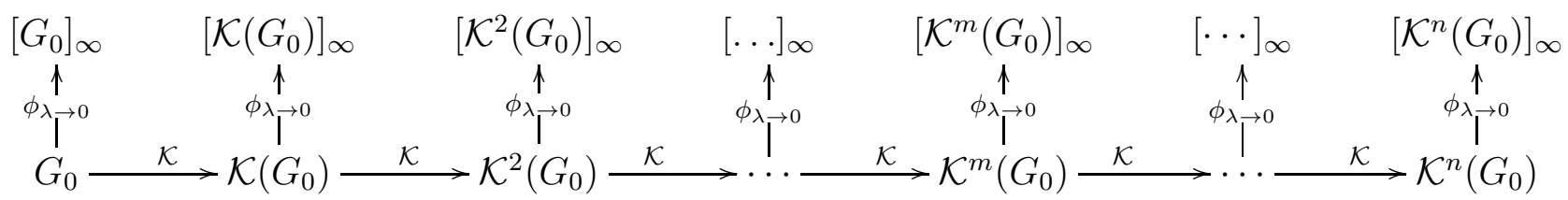

where $\phi_{\lambda \rightarrow 0}$ stands for $\lim _{\lambda \rightarrow 0} \phi_{\lambda}$, and $\mathcal{K}^{i}\left(G_{0}\right)=\underbrace{\mathcal{K}(\mathcal{K}(\ldots \mathcal{K}}_{i \text { times }}\left(G_{0}\right) \ldots)=G_{i}$. The lower horizontal chain contains the discrete spaces that could be strictly quantum or semiclassical and each is derived from the previous one by a coarse graining operation $\mathcal{K}$ that can be a pure quasi-isometry or a rough isometry. The upper horizontal chain contains the continuum limits of the discrete spaces in the lower horizontal chain that are connected to their corresponding discrete spaces by the recaling maps $\phi_{\lambda}$ for $\lambda \rightarrow 0$. We call the lower chain the coarse graining chain, and the upper one the continuum limit chain.

Based on our previous discussions, two cases are now possible. Either the graphs $G_{j}, G_{j+1}=\mathcal{K}\left(G_{j}\right)$, in the coarse graining chain are connected by a pure quasi-isometry $\mathcal{K}$ in which case they are structurally different, or by a rough-isometry $\mathcal{K}$ which means they are structurally similar since their GH-distance is finite. We will now show what this means for their continuum limits. We start by providing a simple lemma,

Lemma IX.6. For $X, X^{\prime}$ being two metric spaces, we have

$$
d_{G H}\left(\lambda X, \lambda X^{\prime}\right)=\lambda \cdot d_{G H}\left(X, X^{\prime}\right) .
$$

Proof. On $X, X^{\prime}, X \sqcup X^{\prime}$ all metrics can be jointly scaled by a factor $\lambda$.

Using this we can see that if in the above coarse graining chain of graphs two members $G_{i}, G_{j}$ have $d_{G H}\left(G_{i}, G_{j}\right)=\infty$, then

$$
d_{G H}\left(G_{i, \infty}, G_{j, \infty}\right)=\infty
$$

i.e., their respective limit spaces also lie in different classes. 
Furthermore, we can see from the above lemma that if we enter the regime where all the spaces $G_{l}$ are roughly isometric, that is, if for two consecutive members $G_{i}, G_{j}$ we have $d_{G H}\left(G_{i}, G_{j}\right)<\infty$, then

$$
d_{G H}\left(G_{i, \infty}, G_{j, \infty}\right)=0
$$

and thus they have the same continuum limit. We argued above that this joint continuum limit space represents the phase cell of microstates which look classically or macroscopically the same.

We will conclude this section with a fundamental observation which sheds some light on our continuum space-time on the various scales of resolution. Furthermore it shows that our above observation concerning the toy model of $\mathbb{Z}^{n} / \mathbb{R}^{n}$ was not accidental. Let us take two elements $G_{i}, G_{j}$ from the coarse graining chain of graphs which are assumed to be only purely quasi-isometric and each having a continuum limit $G_{i, \infty}, G_{j, \infty}$ under the limit $\lambda \rightarrow 0$ or $l \rightarrow \infty$ of the scaling map

$$
\phi_{l}:\left(X, d_{X}\right) \longmapsto\left(X, \lambda d_{X}\right), \quad \lambda=2^{-l} .
$$

Then,

Theorem IX.7. Under the above assumptions, the scaling limits $G_{i, \infty}, G_{j, \infty}$ are homeomorphic topologically but carry different metrics. With the help of the homeomorphism map, the limit spaces can then even chosen to be the same topological space, but carrying different metrics. That is, if our picture of $S-T$ on the various scales is correct, the various scales differ from each other with respect to the metric but live on the same topological space.

For a proof, see appendix B

We would like to add a remark what this means physically. One should note that the different metrics are not artificially imposed from outside but result from the structural differences hidden in the deeper layers of our space-time. That means, they may result for example from the existence of short cuts or microscopic wormholes on certain scales of our coarse graining process, a possibility we mention at the end of the following section.

\section{DIMENSION}

We mentioned the concept of dimension as a characteristic of such discrete and irregular spaces like our graphs/networks in the introduction. It was briefly remarked there why we chose our particular notion, being guided mainly by purely physical motivations [25]. We later realized that our concept is closely related to the notion of growth degree in geometric group theory (see section 4 in [24]).

It turned out that this notion has a lot of stability properties and it is interesting to study its behavior under the geometric RG. In [25] we studied two slightly different versions. Here, we define

$$
D(G)=\lim _{r \rightarrow \infty} \frac{\log \beta\left(G, v_{i}, r\right)}{\log r}
$$

Note that in general only lim sup and lim inf of the right hand side exist, but for convenience, we assume here that instead, its ordinary limit does exist.

Remark X.1. Note that in the cases we study, the value on the left hand side of (10.1) is independent of the reference vertex $v_{i}$ which is one of the stability properties of $D(G)$ as we mentioned above. 
It is of tantamount importance as a characteristic of our limit or continuum spaces, whether $D(G)$ is an integer or some non-integer real value, which indicates the existence of a fractal limit space. We begin by compiling some results we proved in [24]. First we have the important result:

Theorem X.2. If $G_{1}, G_{2}$ are quasi-isometric, with globally bounded vertex degree, then they have the same dimension (theorem 2.22 in [24]).

Conclusion X.3. This implies that in our RG scenario, the dimension of the various spaces remain constant under coarse-graining or scaling limit provided the above assumption is fulfilled. This is then in particular the case for the resulting continuum limits.

It follows that if one wants to have a changing dimension which depends for example on the scale of spatial resolution one has to change these assumptions.

Our macroscopic space-time is four-dimensional. It is a surprisingly deep question in our context whether a general infinite graph/network has an integer dimension. The whole section 3 of [24] was devoted to this problem. This investigation culminated in the following theorem:

Theorem X.4. Let $G$ be a graph with locally finite vertex degree, being connected, and vertex transitive, then its growth degree, and hence its dimension, is an integer. The same holds then for graphs being quasi-isometric to $G$.

That is, if we want to have an integer dimension, one way is to start from such relatively homogeneous graphs (note that vertex transitivity implies a constant vertex degree).

We want to briefly come back to the possibility of changing the dimension under the $\mathrm{RG}$, i.e. the picture that the individual scales of resolution of our S-T may have their own dimensions (possibly non-integer ones on more primordial scales). We started to discuss this possibility in section 8 of [32] where we introduced critical network states. These are states with both a local and a translocal wiring structure. If we have to delete these translocal edges in the course of reconstructing a smooth macroscopic space-time, we may leave the class of quasi-isometries and hence our network dimension may change.

This idea was further explored in [52] where we developed the concept of wormhole spaces. We argued that for example the BH-area law, the holographic principle and quantum entanglement can find a natural explanation in such a framework.

\section{CONCLUSION}

In this work, we have laid out the basis of a novel approach to the emergence of smooth spacetime from a discrete substratum based on purely geometric notions. The approach resembles the Wilsonian renormalization procedure. The starting point or the initial condition is a metric space $\left(G_{0}, d_{0}\right)$, where $G_{0}$ is an uncolored graph 11 and $d_{0}$ a graph metric defined on it. The renormalization procedure contains two operations: a coarse graining operation

$$
\mathcal{K}: G_{i} \rightarrow G_{i+1}
$$

${ }^{11}$ We postpone the treatment of colored graphs to a future work. 
that is a quasi-isometry map between discrete spaces, and a rescaling map

$$
\phi_{\lambda}:\left(G_{i}, d_{i}\right) \rightarrow\left(G_{i}, \lambda d_{i}\right)
$$

whose limit $\lambda \rightarrow 0$ yields the continuum limit $\left(G_{i, \infty}, d_{i, \infty}\right)$ of the discrete space $\left(G_{i}, d_{i}\right)$,

$$
\lim _{\lambda \rightarrow 0} \phi_{\lambda}\left(\left(G_{i}, d_{i}\right)\right)=\lim _{\lambda \rightarrow 0}\left(G_{i}, \lambda d_{i}\right)=\left(G_{i, \infty}, d_{i, \infty}\right) .
$$

Here the parameter $\lambda$, parametrizes the distance between the points on the different length scales. Combining these two operations, we represent again our findings in the following graphic.

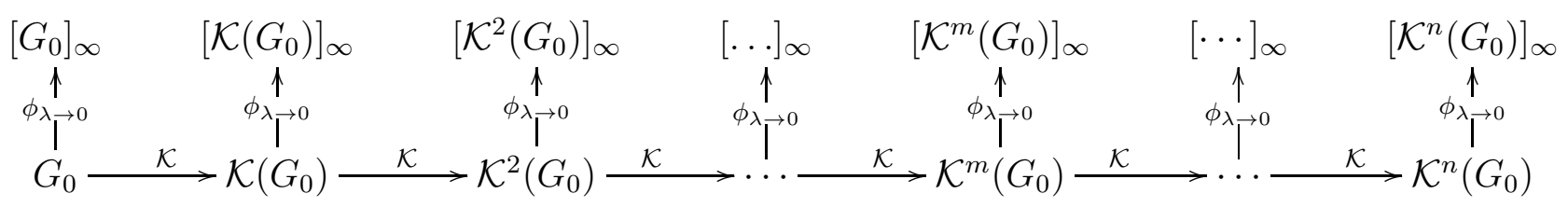

where $\left[\mathcal{K}^{i}\left(G_{0}\right)\right]_{\infty}=G_{i, \infty}$. The lower chain consisting of discrete coarse grained spaces is called the coarse graining chain while the upper one is the continuum limit chain.

Our coarse graining operations, $\mathcal{K}$, lie in the class of quasi-isometries. We show that in the case, where two consecutive members of the coarse chain $G_{i}$ and $G_{i+1}$ are related by a true quasi-isometry, their continuum limits $G_{i, \infty}, G_{i+1, \infty}$ carry different metrics, so their Gromov-Hausdorff $(\mathrm{GH})$ distance, $d_{G H}$, is infinite

$$
d_{G H}\left(G_{i, \infty}, G_{i+1, \infty}\right)=\infty .
$$

This distance is a measure of how much two spaces are (non)isometric, and an infinite distance tells us that they are structurally distinct. However, we show that, although they carry different metrics, they are topologically homeomorphic, and can even be chosen to be the same topological space. This implies that different levels of spacetime will have different metric even if they are the same set. As to the physical implications see the remarks at the end of section [X]

The coarse graining operation goes on until one of the two cases (or both) happen: either $\mathcal{K}$ turns into a rough isometry, or the sequence of graphs in the coarse graining chain reaches a stable fixed point or a set of accumulation points already on the discrete scale.

In the former case, we show that the continuum limits of these roughly isometric spaces are the same, in the sense that their GH-distance is zero, $d_{G H}\left(G_{i, \infty}, G_{i+1, \infty}\right)=0$. This means that they are isometric. We identify these roughly isometric spaces with the phase cell of our state space which is the basin of attraction for the corresponding continuum limit and which we associate (in the optimal case) with our classical space-time.

The latter case happens if the sequence of coarse grained spaces are uniformly compact, in which case, we can use the Gromov's compactness theorem to show their convergence with respect to $d_{G H}$. We think however that this possibility is not the generic one.

In any case, we show that the continuum limits are scale invariant, in the sense that under the rescaling map

$$
\phi_{\lambda}:\left(G_{i, \infty}, d_{i, \infty}\right) \rightarrow\left(G_{i, \infty}, \lambda d_{i, \infty}\right)
$$

their GH-distance is zero,

$$
d_{G H}\left(X_{\infty}, l X_{\infty}\right)=0 .
$$


Finally we present a relevant notion of dimension for these discrete spaces and very briefly discuss its properties. It turns out that under certain conditions, such as graphs of locally finite vertex degree, being connected and vertex transitive, not only this dimension is an integer, but is also stable (i.e. invariant) under both $\mathcal{K}$ and $\phi_{\lambda}$. However, if we deal with graphs/networks having both a local and translocal wiring structure it may happen that the coarse graining procedure is no longer local in the sense defined above. In that case the dimension may become dependent on the coarse graining scale so that each scale may have its own dimension (cf. the remarks at the end of section $\mathrm{X}$ ).

\section{ACKNOWLEDGMENTS}

The authors would like to thank the anonymous referee whose remarks and suggestions helped to improve the typescript. S. R. would like to acknowledge the partial support of CONACyT Grant No. 237351: Implicaciones Físicas de la Estructura del Espaciotiempo. He also would like to acknowledge the support of the PROMEP postdoctoral fellowship (through UAM-I) and the grant from Sistema Nacional de Investigadores of CONACyT.

\section{Appendix A: Some relevant definitions}

Definition A.1. A pseudometric on a set $S$ is a map $d_{S}: S \rightarrow \mathbb{R}$ which has all the properties of the metric except the property that $d_{S}\left(x_{1}, x_{2}\right)=0 \Leftrightarrow x_{1}=x_{2}$ for all $x_{1}, x_{2} \in S$.

Definition A.2. A metric space in which every sequence has a subsequence that converges to a point in $M$ is called sequentially compact. For metric spaces this is equivalent to the compactness defined via open covers.

Definition A.3. A metric space is called proper if all its closed balls, $B(x, r), \forall x \in M$, are compact.

Definition A.4. A proper metric space is locally compact if every point has a compact neighborhood.

Corollary A.5. Proper spaces are locally compact, but the converse is not true in general.

Definition A.6. A metric space $M$ is complete iff every Cauchy sequence has a limit in $M$.

Definition A.7. A metric space $M$ is bounded if there exists some number $r$, such that $d(x, y) \leq r, \forall x, y \in M$. The smallest possible such $r$ is called the diameter of $M$.

Definition A.8. A metric space $M$ is precompact or totally bounded if for every $r>0$ there exist finitely many open balls of radius $r$ whose union covers $M$.

\section{Appendix B: Proof of some of the theorems}

\section{Theorem V.3}

In a slightly different context a related result was already proved in section VII of [32]. It is also contained in [50]. The order of a clique is bounded by $\operatorname{deg}(G)+1$. The cliques, containing 
a fixed vertex, $v_{0}$, are lying in its 1-neighborhood $B\left(v_{0}, 1\right)$, they hence consist of subsets of $B\left(v_{0}, 1\right)$. Furthermore, no clique is contained in another clique (due to maximality). They hence represent a Sperner system (see e.g. [53]) and it follow 12 .

$$
\#\left(\text { cliques } \mid v_{0} \in \text { clique }\right) \leq\left(\begin{array}{c}
\operatorname{deg}(G)+1 \\
\lfloor(\operatorname{deg}(G)+1) / 2\rfloor
\end{array}\right)=: \operatorname{Sper}_{G} \text {. }
$$

We hence get that the vertex degree in $\mathcal{C}(G)$ is bounded by

$$
(\operatorname{deg}(G)+1) \cdot\left(\operatorname{Sper}_{G}-1\right) .
$$

We now discuss the rough isometry between $G$ and $\mathcal{C}(G)$. In a first step we define the map

$$
f: G \longrightarrow \mathcal{C}(G)
$$

For each vertex $v$ there exist cliques which contain $v$. We choose one of them as $f(v)$. Let $C$ be an arbitrary clique in $\mathcal{C}(G)$. It contains a vertex $v$ and we hence have

$$
v \in f(v) \cap C \neq \emptyset \text {. }
$$

It follows that $\mathcal{C}(G)$ is contained in the one-neighborhood of $f(G)$ :

$$
\mathcal{C}(G) \subset U_{1}(f(G))
$$

Now let $v, v^{\prime}$ be two arbitrary vertices in $G$. It exists a (geodesic) path, $\gamma$, from $v$ to $v^{\prime}$ having a length $l(\gamma)=d\left(v, v^{\prime}\right)$. Each pair, $\left(v_{j-1}, v_{j}\right), j=1, \ldots, d\left(v, v^{\prime}\right)$, in the path lies in a clique $C_{j}$. The consecutive cliques $C_{j}, C_{j+1}$ have non-void overlap, i.e., they are connected by an edge in $\mathcal{C}(G)$. We hence get a path in $\mathcal{C}(G)$ of length $d\left(v, v^{\prime}\right)-1$.

It follows that $f(v), f\left(v^{\prime}\right)$, which each can have at most distance 1 from the initial vertex $v$ or end vertex $v^{\prime}$, have distance at most $d\left(v, v^{\prime}\right)+1$. On the other hand, by the same token we can conclude:

$$
d\left(f(v), f\left(v^{\prime}\right) \geq d\left(v, v^{\prime}\right)-1,\right.
$$

as a path, $\gamma^{\prime}$ in $\mathcal{C}(G)$ between $f(v), f\left(v^{\prime}\right)$ of length $l\left(\gamma^{\prime}\right) \leq d\left(v, v^{\prime}\right)-2$ yields a path of length $d\left(v, v^{\prime}\right)-1$ in $G$, which is a contradiction. We finally have:

$$
d\left(v, v^{\prime}\right)-1 \leq d\left(f(v), f\left(v^{\prime}\right)\right) \leq d\left(v, v^{\prime}\right)+1
$$

which proves our theorem.

\section{Theorem IX.7}

The proof is given in several steps which may be useful for their own sake. So let $G_{i}, G_{j}$ be $(\lambda, \epsilon)$-quasi-isometric with scaling limits $G_{i, \infty}, G_{j, \infty}$. This implies that there exists a map $f: G_{i} \rightarrow G_{j}$ with $d_{G_{j}}\left(y, f\left(G_{i}\right) \leq \epsilon\right.$ for all $y \in G_{j}$. We then have

Lemma B.1. $G_{j}$ and $f\left(G_{i}\right)$ are roughly isometric.

$\overline{{ }^{12} \text { In [32] }}$ we provided a simpler but cruder bound. 
Proof. It holds

$$
d_{G_{j}}\left(y_{1}, y_{2}\right) \leq d_{G_{j}}\left(y_{1}, f\left(x_{1}\right)+d_{G_{j}}\left(f\left(x_{1}\right), f\left(x_{2}\right)\right)+d_{G_{j}}\left(f\left(x_{2}\right), y_{2}\right)\right.
$$

for certain elements $x_{1}, x_{2}$. The RHS is $\leq 2 \epsilon+d_{G_{j}}\left(f\left(x_{1}\right), f\left(x_{2}\right)\right)$. We now choose a map from $y \in G_{j}$ to a corresponding $f(x)$ for a suitable $x$ which defines an $\epsilon$-rough isometry.

Corollary B.2. It follows that $G_{j}, f\left(G_{i}\right)$ have the same continuum limit.

For the $x, x^{\prime}$ which are mapped on the same $y$ under the quasi-isometry $f$ we have

$$
0 \geq \lambda^{-1} d_{G_{i}}\left(x, x^{\prime}\right)-\epsilon \text { hence } \quad d_{G_{i}}\left(x, x^{\prime}\right) \leq \lambda \cdot \epsilon
$$

We choose a quasi-inverse $g$ to $f$ by selecting one element in the preimage of $y$. We observe Observation B.3. $G_{i}$ is also roughly isometric to $g\left(f\left(G_{i}\right)\right)$.

Conclusion B.4. We can restrict ourselves to the spaces $f\left(G_{i}\right), g\left(f\left(G_{i}\right)\right)$. The quasiisometry defines a (bilipschitzian) equivalence

$$
\lambda^{-1} d_{G_{i}}\left(x, x^{\prime}\right) \leq d_{G_{j}}\left(f(x), f\left(x^{\prime}\right)\right) \leq \lambda d_{G_{i}}\left(x, x^{\prime}\right)
$$

between the two spaces.

Now we take the scaling limit on both sides and get

$$
2^{-l} \cdot \lambda^{-1} d_{G_{i}}\left(x, x^{\prime}\right) \leq 2^{-l} \cdot d_{G_{j}}\left(f(x), f\left(x^{\prime}\right)\right) \leq 2^{-l} \cdot \lambda d_{G_{i}}\left(x, x^{\prime}\right)
$$

with corresponding bijective maps $f_{l}: g\left(f\left(G_{i}\right)\right) \rightarrow f\left(G_{i}\right)$. We know that $2^{-l} G_{i}, 2^{-l} G_{j}$ converge in GH-sense to $G_{i, \infty}, G_{j, \infty}$. By theorem VI.3 and the following remarks we conclude that there exist rough isometies between $2^{-l} G_{i}, 2^{-l} G_{j}$ and $G_{i, \infty}, G_{j, \infty}$. Equation (B11) shows that, in the scaling limit, we get a continuous bijective map $f_{\infty}$ between $G_{i, \infty}, G_{j, \infty}$ with metrics $d_{i, \infty}, d_{j, \infty}$ according to the bilipschitzian equivalence, described above. This shows that the two limit spaces are homeomorphic. The following observation concludes the proof.

Observation B.5. With the help of the map $f_{\infty}$ we can transfer the metric structure from $G_{i, \infty}$ to $G_{j, \infty}$ and get two metrics on the same space which are related to each other by the above bilipschitzian equivalence which ultimately is a consequence of the quasi-isometry we started from.

A related result was proved in [50] by different methods.

[1] T. Thiemann, Modern Canonical Quantum General Relativity (Cambridge University Press, 2008).

[2] C. Rovelli and F. Vidotto, Covariant Loop Quantum Gravity: An Elementary Introduction to Quantum Gravity and Spinfoam Theory (Cambridge University Press, 2014).

[3] J. C. Baez, "Spin networks in nonperturbative quantum gravity," Proc. AMS on Knots and Physics (1995), gr-qc/9504036. 
[4] S. Kauffman and L. Smolin, "Combinatorical dynamics in quantum gravity," in Towards Quantum Gravity, Vol. SLN Phys. 2000, edited by J. Kowalski-Glikman (Proc. Polonica, Poland, 1999) hep-th/9809161.

[5] F. Markopoulou, "An algebraic approach to coarse graining," (2000), hep-th/0006199.

[6] F. Markopoulou and L. Smolin, "Causal evolution of spin networks," Nucl. Phys. B 508, 409 (1997), gr-qc/9702025.

[7] D. Oriti, "Spacetime geometry from algebra," Rep. Prog. Phys. 64, 1703 (2001), gr-qc/0106091.

[8] A. Perez, "Spin foam models for quantum gravity," Class. Quantum Grav. 20, 1243 (2003).

[9] T. Konopka, F. Markopoulou, and L. Smolin, "Quantum graphity," (2006), hep-th/0611197.

[10] T. Konopka, "Statistical mechanics of graphity models," Phys. Rev. D 78, 044032 (2008).

[11] B. Dittrich, "The continuum limit of loop quantum gravity," (2014), 1409.1450.

[12] B. Dittrich, E. Schnetter, C. J. Seth, and S. Steinhaus, "Coarse graining flow of spin foam intertwiners," (2016), 1609.02429.

[13] D. Oriti, "The microscopic dynamics of quantum space as a group field theory," in Foundations of Space and Time: Reflections on Quantum Gravity, edited by J. Murugan G. Ellis and A. Weltman (Cambridge University Press, Cambridge, 2012) 1110.5606.

[14] H. Chen, N. Sasakura, and Y. Sato, "Emergent classical geometries on boundaries of randomly connected tensor networks," (2016), 1601.04232.

[15] D. Oriti, "Disappearance and emergence of space and time in quantum gravity," (2013), 1302.2849.

[16] J. Ambjorn, A. Goerlich, J. Jurkiewicz, and R. Loll, "Nonperturbative quantum gravity," Phys. Rep. 519, 127-210 (2012), 1203.3591.

[17] M. Requardt and S. Rastgoo, "The structurally dynamic cellular network and quantum graphity approaches to quantum gravity and quantum geometry - a review and comparison," Journal of Cellular Automata (5-6), 341-392, 1501.00391.

[18] C.A.Trugenberger, "Combinatorial quantum gravity," (2016), 1610.05934.

[19] G. Bianconi, "Interdisciplinary and physics challenges of network theory," Europhysics Letters 111 (2015), 1509.00345

[20] M. Requardt, "Scale free small world networks and the structure of quantum space-time," (2003), gr-qc/0308089.

[21] C. W. Misner, K. S. Thorne, and J. A. Wheeler, Gravitation (W. H. Freeman, 1973).

[22] L. Bombelli, A. Corichi, and O. Winkler, "Semiclassical quantum gravity: Obtaining manifolds from graphs," Class. Quantum Grav. 26 (2009), 0905.3492.

[23] M. Requardt, "Cellular networks as models for planck-scale physics," J. Phys. A 31, 7997 (1998), hep-th/9806135.

[24] M. Requardt, "The continuum limit of discrete geometries," Int. J. Geom. Meth. Mod. Phys. 3, 285 (2006).

[25] T. Nowotny and M. Requardt, "Dimension theory on graphs and networks," J. Phys. A 31, 2477 (1998), hep-th/9707082.

[26] M. Requardt and S. Roy, "(quantum) spacetime as a statistical geometry of fuzzy lumps and the connection with random metric spaces," Class. Quantum Grav. 18, 3039 (2001), 003117.

[27] P. A. Schilpp, Albert Einstein: Philospher-Scientist (Cambridge University Press, 1949).

[28] M. Seriu, "Space of spaces as a metric space," Commun. Math. Phys. 29, 393-405.

[29] A. Ashtekar and P. Singh, "Loop quantum cosmology: a status report," Class. Quantum Grav. 28, 213001 (2011), 1108.0893.

[30] M. Requardt, "(quantum) space-time as a statistical geometry of lumps in random networks," 
Class. Quantum Grav. 17, 5588 (2000), gr-qc/9912059.

[31] M. Requardt, "Graph-laplacians and dirac operators on (infinite) graphs and the calculation of the connes-distance-functional," J. Phys. A: Math. Gen. 35, 759 (2000), math-ph/0001026v3.

[32] M. Requardt, "A geometric renormalization group and fixed point behavior in discrete quantum space-time," J. Math. Phys. 44, 5588 (2003), gr-qc/0110077.

[33] T. Nowotny and M. Requardt, "Emergent properties in structurally dynamic disordered cellular networks," J. Cell. Automata 2, 273-289 (2007), cond-mat/0611427.

[34] M. Requardt, "Observables need not be diffeomorphism invariant in classical and quantum gravity," (2003), 1206.0832 .

[35] J. v. Neumann, "Beweis des ergodensatzes und des h-theorems in der neuen mechanik," Zeitschr.Phys. 57, 30 (1929).

[36] S. Goldstein, J. L. Lebowitz, R. Tumulka, and N. Zhangi, "Long-time behavior of macroscopic quantum systems: Commentary accompanying the english translation of john von neumann's 1929 article on the quantum ergodic theorem," European Phys. J. H35, 173-200 (2010), 1003.2129 .

[37] M. Requardt, "An alternative to decoherence by environment and the appearance of a classical world," (2010), 1009.1220.

[38] R. Gurau, "Invitation to random tensors," SIGMA 12, 094 (2016), 1609.06439.

[39] F. Anzà and G. Chirco, "Quantum typicality in spin network states of quantum geometry," (2016), 1605.04946.

[40] S. Goldstein, J. L. Lebowitz, C. Mastrodonato, R. Tumulka, and N. Zhangi, "Normal typicality and v.neumann's quantum ergodic theorem," Proc. Roy. Soc. A466, 3203-3224 (2010), 0907.0108 .

[41] M. Ledoux, The Concentration of Measure Phenomenon (American Mathematical Society, N.Y., 2001).

[42] M. Gromov, Metric Structures for Riemannian and Non-Riemannian Spaces (Birkhaeuser, N.Y., 1998).

[43] P. de la Harpe, Topics in Geometric Group Theory (University of Chicago Press, 2000).

[44] M. R. Bridson and A. Haefliger, Metric Spaces of Non-Positive Curvature (Springer N.Y., 1999).

[45] B. Bollobas, Random Graphs, 2nd ed. (Cambridge University Press, 2001).

[46] M. A. Pisana, "The icosahedron is clique divergent," Discr. Math. 262, 229 (2003).

[47] M. A. Pisana, "Distances and diameters on iterated clique graphs," Discr. Appl. Math. 141, 225 (2004).

[48] G. A. Edgar, Measure, Topology, and Fractal Geometry (Springer, N.Y., 1990).

[49] P. Petersen, Gromov-Hausdorff convergence of metric spaces, Vol. 54 (AMS Proc. Pure Math., 1993) p. 489.

[50] A. Lochmann, PhD Thesis, Ph.D. thesis, University of Goettingen (2005).

[51] M. Gromov, "Groups of polynomial growth and expanding maps," Publ. Math. IHES 53, 53 (1981).

[52] M. Requardt, "Wormhole spaces: The common cause for the black hole entropy-area law, the holographic principle and quantum entanglement," 0910.4017.

[53] B. Bollobas, Combinatorics (Cambridge University Press, 1994). 